\title{
AUTOMORPHISMS OF TRANSFORMATION SEMIGROUPS
}

\author{
by S. P. FITZPATRICK and J. S. V. SYMONS \\ (Received 18th December 1973)
}

\section{Introduction}

It is common property in the theory of transformation semigroups that the presence of all the constant maps ensures that automorphisms are induced by a permutation of the underlying set. Essentially, this goes back to Malcev (2); it has been extensively generalised by Sullivan in (4). For semigroups which do not contain the constants (for example, all surjective transformations of a set, or all injections) there is, as yet, no similar result. The purpose of this note is to provide one.

\section{Results}

The notation and terminology of (1) is used. Our result is:

Theorem. If $S$ is a transformation semigroup over an infinite set $X$, and $S$ contains $\mathscr{G}_{X}$, the group of all permutations of $X$, then the automorphisms of $S$ are inner, that is, of the form

for fixed $g$ in $\mathscr{G}_{X}$.

$$
\alpha \rightarrow g^{-1} \alpha g \quad(\alpha \in S)
$$

We now prove this result. Let $S$ be as stated and $\phi$ be an automorphism of $S$. Clearly $\phi$ induces an automorphism of $\mathscr{G}_{X}$, for elements of this set are characterised in $S$ by the existence of two-sided inverses. Thus by a well-known property of $\mathscr{G}_{X}$ (see (3)), we have

$$
h \phi=g^{-1} h g \quad\left(h \in \mathscr{G}_{X}\right)
$$

for some fixed $g$ in $\mathscr{G}_{X}$. Let us replace $\phi$ by $\phi \circ \psi$ where $\psi$ is the automorphism

$$
\alpha \rightarrow g \alpha g^{-1} \quad(\alpha \in S) \text {. }
$$

Then $\phi$ now fixes each map in $\mathscr{G}_{X}$ and it is enough to show that such an automorphism is trivial.

We first dispense with the case where $S$ consists entirely of injections. We derive this from the following, the proof of which is straightforward.

Lemma. If $a, b, c$, d are elements of $X$ with $a \neq b$ and $c \neq d$, and if $\alpha$ is one to one then:

$$
(a, b) \alpha(c, d)=\alpha \quad \text { if and only if }\{a, b\} \alpha=\{c, d\}
$$


(Here we are using $(a, b)$ to denote the two cycle interchanging $a$ and $b$.) The invariance of the first equality under automorphisms fixing permutations shows that

$$
\{a, b\} \alpha=\{a, b\} \alpha \phi
$$

whenever $a \neq b$. Now if $a, b$ and $c$ are all distinct, as required.

$$
\{a \alpha\}=\{a, b\} \alpha \cap\{a, c\} \alpha=\{a, b\} \alpha \phi \cap\{a, c\} \alpha \phi=\{a \alpha \phi\}
$$

Henceforth we assume that $S$ contains a mapping $\alpha$ that is not one to one. It follows that we may assume further each mapping in $S$ has infinite rank. For, if $\beta$ has finite rank we consider the sequence of elements of $S$

$$
\beta_{n}=\beta g_{1} \alpha g_{2} \ldots g_{n} \alpha,
$$

where each $g_{i+1}$ is a permutation mapping two elements of the range $\beta_{i}$ to two elements identified by $\alpha$. Eventually this process yields a constant map, $\kappa$, say. But then $\kappa \mathscr{G}_{X}$ is contained in $S$ and this is the set of all constants. In this case the result follows from the "common property" alluded to in the introduction.

The following fact is so essential to our result that we state it formally.

Lemma. If $\pi_{\alpha}$ denotes the equivalence on $X$ induced by $\alpha$ in $S$ then $\pi_{\alpha}=\pi_{\alpha \phi}$.

Proof. It is enough to note that

$$
x \pi_{\alpha} y \Leftrightarrow(x, y) \circ \alpha=\alpha \Leftrightarrow(x, y) \circ \alpha \phi=\alpha \phi \Leftrightarrow x \pi_{\alpha \phi} y .
$$

Now let $A$ and $B$ be partition classes of $\pi_{a}$, equally, $\pi_{\alpha \phi}$. For distinct $a$ and $b$ in $X$ we denote by $P(a, b, \alpha)$ the statement:

$$
\text { for each } \beta \text { in } S, a \pi_{\beta} b \text { implies }(A \cup B) \pi_{\alpha \beta}(A \cup B) \text {. }
$$

We assert that $P(a, b, \alpha)$ is necessary and sufficient for

$$
\{A \alpha, B \alpha\}=\{a, b\} \text {. }
$$

It is clear that $Q$ implies $P$. On the other hand if $Q$ fails, we select $\beta$ in $S$ with the following properties

$$
a \beta=b \beta ;(A \alpha) \beta \neq(B \alpha) \beta .
$$

(To obtain such a map in $S$ we select $\delta$ in $S$ which is not one to one. We next choose $g$ in $\mathscr{G}_{X}$ so that $\{a g, b g\}$ is contained in a single $\pi_{\delta}$-class, but $(A \alpha) g$ and $(B \alpha) g$ lie in different $\pi_{\delta}$-classes. This uses the fact that $\mathscr{G}_{X}$ is multiply transitive. The required $\beta$ is now $g \delta$.) For such a $\beta$ we have $a \pi_{\beta} b$, but $A \alpha \beta \neq B \alpha \beta$.

We have shown that $(P)$ and $(Q)$ are equivalent statements. However, it is easy to see from the Lemma that $P(a, b, \alpha)$ is equivalent to $P(a, b, \alpha \phi)$. We conclude that

$$
\{A \alpha, B \alpha\}=\{a, b\}=\{A \alpha \phi, B \alpha \phi\} .
$$

It follows that $A \alpha=A \alpha \phi$, and hence that $\alpha=\alpha \phi$. The proof of the theorem is complete. 


\section{AUTOMORPHISMS OF TRANSFORMATION SEMIGROUPS 329}

If $X$ is finite, the theorem still holds with an exception when $S$ is the symmetric group on six objects. To see this, observe that if $X$ is finite and $S$ contains $\mathscr{G}_{X}$, then $S$ either coincides with $\mathscr{G}_{X}$ or else contains a map which is not one to one. In the latter case $S$ contains a constant map, hence all, and the result follows from "common property". In the former case there is only one full symmetric group with an outer automorphism, $S_{6}$.

\section{REFERENCES}

(1) A. H. Clifford and G. B. Preston, The Algebraic Theory of Semigroups, Vols. I and II (American Math. Soc., Providence, Rhode Island, 1961 and 1967).

(2) A. I. MALCEv, Symmetric groupoids, Math. Sb. (N.S.), 31 (73) (1952), 136-151.

(3) W. R. Scort, Group Theory (Prentice-Hall, 1964).

(4) R. P. Sullivan, Automorphisms of Transformation semigroups (to appear).

UNIVERSITY OF WESTERN AUSTRALIA

NEDLANDS, 6009 